\title{
Central Venoplasty in Patients with Cardiac Implantable Electronic Devices
}

\author{
Mohammed Aljarie ${ }^{1} \quad$ Mohammed Alahmari ${ }^{1}$ \\ ${ }^{1}$ Division of Vascular and Interventional Radiology, Department of \\ Medical Imaging, Ministry of National Guard - Health Affairs; King \\ Abdullah International Medical Research Center, King Saud bin \\ Abdulaziz University for Health Sciences, Riyadh, Saudi Arabia
}

\begin{abstract}
Address for correspondence Mohammed Aljarie, Division of Vascular and Interventional Radiology, Department of Medical Imaging, Ministry of National Guard - Health Affairs; King Abdullah International Medical Research Center, King Saud bin Abdulaziz University for Health Sciences, Riyadh-11481, Saudi Arabia (e-mail: aljariemo@gmail.com).
\end{abstract}

\begin{abstract}
Keywords

- balloon angioplasty

- dialysis

- Occlusion

- pacemaker

- stenosis

Objective The aim of this study was to assess the safety and effectiveness of the central venous angioplasty in patients with central venous occlusion and cardiac implantable electronic device (CIED) without lead extraction.

Materials and Methods A retrospective study was used to evaluate the effectiveness of 37 central venous angioplasty procedure for 15 patients with CIED without lead extraction.

Results Technical success was achieved in $97 \%(n=36 / 37)$ and clinical success was achieved in $89 \%(33 / 37)$ of the procedures. One procedure failed recanalization of chronic total occlusion of the left subclavian vein, and the patient required fistula embolization due to severe arm swelling. Another procedure failed initially to recanalize long-segment occlusion involving the right subclavian vein/brachiocephalic vein and superior vena cava in a patient with a history of Hickman line and left-sided CIED. This was successfully recanalized and angioplastied on a subsequent session. No lead fracture or dislodgment was documented in any procedure. No procedure-related complication was documented within 2 weeks after the angioplasty. Six-month primary patency was achieved in $62 \%(23 / 37)$ of the procedures. Ten patients $(66 \%)$ required an average of 1.4 reinterventions (range: $1-4$ interventions) during the follow-up time with mean time to reintervention of 318 days (5-1,380 days). Two patients required early reinterventions within 10 days due to catheter dysfunction.

Conclusion Findings of this study support the existing evidence on the safety and effectiveness of balloon angioplasty without lead extraction.
\end{abstract}

\section{Introduction}

The incidence of central venous occlusion (CVO) after placement of cardiac implantable electronic devices (CIED) ranges between 13 and 64\%. However, symptomatic venous stenosis is seen in less than $3 \%$ of patients due to presence of well-developed collaterals. ${ }^{1-5}$

In contrary, $70 \%$ patients with CIED and ongoing hemodialysis using arteriovenous fistula or tunneled dialysis catheters may develop symptoms related to central venous stenosis or published online June 4, 2021
DOI https://doi.org/

$10.1055 / \mathrm{s}-0041-1730121$ ISSN 2542-7075 (c) 2021. The Pan Arab Interventional Radiology Society

This is an open access article published by Thieme under the terms of the Creative Commons Attribution-NonDerivative-NonCommercial-License, permitting copying and reproduction so long as the original work is given appropriate credit. Contents may not be used for commercial purposes, or adapted, remixed, transformed or built upon. (https://creativecommons.org/licenses/by-nc-nd/4.0/).

Thieme Medical and Scientific Publishers Private Ltd. A-12, Second Floor, Sector -2, NOIDA -201301, India 
occlusion. The combination of increased venous flow and the coexisting venous stenosis secondary to the CIED and dialysis catheter leads to the clinical manifestations of venous hypertension. Endovascular management of CVO related to CEID is increasingly being utilized and reported in the literature. ${ }^{6}$ Despite the theoretical concerns related to possible device malfunction and leads integrity, balloon angioplasty and stent placement are reportedly safe and effective with no device-specific complications. However, data with long-term follow-up remain scarce and largely retrospective in nature..$^{1,6-15}$ This study aims to report our institutional experience with balloon angioplasty for CEID-related CVO in dialysis patients without lead extraction.

\section{Materials and Methods}

This retrospective study was approved by the institutional review board and informed consent was waived. This study aimed at evaluating the safety and effectiveness of central venous balloon angioplasty interventions in the presence of CIED leads.

Between April 2014 and September 2020, 1,500 venous angioplasty and dialysis interventions were reviewed. A total of 37 central venous balloon angioplasty interventions in 15 patients with CIEDs were included in this analysis. Patients' cardiac demographics are displayed in - Table 1.

The mean age was 71 years (56-81 years), with left-sided device in $93 \%$ (14/15 patients), inserted via axillary vein in $47 \%$ (7/15) and subclavian vein in 53\% (8/15). The patient with right-sided device inserted via subclavian vein.

Fourteen patients (93\%) were on dialysis using fistula $(n=5)$ and tunneled dialysis catheter $(n=9)$; while one patient had central venous stenosis related to peripherally inserted central catheter and tunneled Hickman catheter. Detailed patients' demographics and characteristics are illustrated in - Table 2 .
Thirty procedures (81\%) were done due to stenosis, and seven interventions (19\%) were done for total venous occlusion. Procedures were performed for different indications: dialysis catheter dysfunction $(n=18 / 37)$, fistula dysfunction ( $n=14 / 37)$, limb swelling $(n=7 / 37)$ superior vena cava (SVC) syndrome $(n=1 / 37)$, and facial swelling $(n=1 / 37)$.

Venous stenoses were located in SVC alone (35\%), subclavian with brachiocephalic (22\%), brachiocephalic alone (13\%), SVC with brachiocephalic (11\%), subclavian alone ( $8 \%$ ), SVC with brachiocephalic and subclavian (8\%), and SVC with subclavian (3\%).

Angioplasty procedures were done by several interventional radiologists with 1 to 15 years of experience. All procedures were done using balloon angioplasty using various brands and pressures (progressive increments in size with maximum diameter $14 \mathrm{~mm}$ in SVC). No stents were implanted and no leads were extracted at the time of interventions.

Technical success was defined as the ability restore venous patency with less than $30 \%$ residual luminal narrowing with disappearance of venous collaterals or successful disruption of the fibrin sheath with restoration of catheter function. Clinical success was defined as restoration of catheter/fistula function or resolution of venous hypertension symptoms for at least 2 weeks post the procedure. The integrity and position of CIED leads after the procedure were checked on final procedural images. No specific follow-up protocol or scheduled CIED interrogation was recommended.

Time-to-reintervention and patency at last follow-up were recorded, in addition to postprocedural cardiac and noncardiac complications within 2 weeks interval.

\section{Results}

Technical success was achieved in 97\% ( $n=36 / 37)$

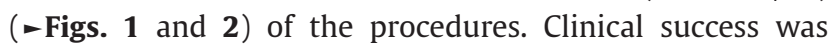
achieved in $89 \%$ (33/37) of the procedures. One procedure

Table 1 Patient's cardiac demographics

\begin{tabular}{|l|l|l|l|l|l|}
\hline Case & CIED type & Manufacturer & Side & No. of leads & Indication for CIED placement \\
\hline 1 & Pacemaker & St. Jude & L & 2 & Complete heart block \\
\hline 2 & Pacemaker & Medtronic & L & 2 & Complete heart block \\
\hline 3 & Pacemaker & St. Jude & L & 2 & Complete heart block \\
\hline 4 & Pacemaker & Medtronic & R & 2 & recurrent syncope \\
\hline 5 & Pacemaker & Medtronic & L & 1 & Atrioventricular block 2nd degree \\
\hline 6 & Pacemaker & Medtronic & L & 1 & Recurrent syncope \\
\hline 7 & Pacemaker & St. Jude & L & 1 & Complete heart block \\
\hline 8 & Pacemaker & St. Jude & L & 2 & Sick sinus syndrome \\
\hline 9 & Pacemaker & Medtronic & L & 2 & Complete heart block \\
\hline 10 & Pacemaker & Medtronic & L & 2 & Sick sinus syndrome \\
\hline 11 & Pacemaker & St. Jude & L & 1 & Atrioventricular block 2nd degree \\
\hline 12 & Defibrillator & St. Jude & L & 3 & Ischemic cardiomyopathy and LBBB \\
\hline 13 & Pacemaker & Medtronic & L & 2 & Atrioventricular block 2nd degree \\
\hline 14 & Pacemaker & Medtronic & L & 1 & Sick sinus syndrome \\
\hline 15 & Defibrillator & Medtronic & L & 1 & Recurrent syncope \\
\hline
\end{tabular}

Abbreviations: CIED, cardiac implantable electronic device; LBBB, left bundle branch block. 
Table 2 Patient's demographic and clinical characteristics

\begin{tabular}{|l|l|l|l|l|l|}
\hline Case & Age & Sex & Vein involved & Presenting symptoms & Comorbidity \\
\hline 1 & 71 & M & SCV + BC & Dysfunctional fistula & CHF \\
\hline 2 & 74 & F & SCV & Dysfunctional fistula & DM, HTN \\
\hline 3 & 66 & F & SCV + BC & Dysfunctional fistula, catheter dysfunction, upper limb swelling & No \\
\hline 4 & 66 & M & SCV + BC & Dysfunctional fistula & DM, HTN \\
\hline 5 & 73 & M & BC & Catheter dysfunction, upper limb swelling & DM, HTN, \\
hypothyroidism
\end{tabular}

Abbreviations: BC, brachiocephalic vein; CHF, congestive heart failure; DM, diabetes mellitus; HTN, hypertension; SCV, subclavian vein; SVC, superior vena cava.

failed recanalization of chronic total occlusion of the left subclavian vein, and the patient required fistula embolization due to severe arm swelling. Another procedure failed initially to recanalize long segment occlusion involving the right subclavian vein/brachiocephalic vein $(\mathrm{SCV} / \mathrm{BCV})$ and SVC in a patient with history of Hickman line and left-sided CIED. This was successfully recanalized and angioplastied on a subsequent session.

No lead fracture or dislodgment was documented in any procedure. Electrocardiographic (ECG) changes occurred in two patients within 2 weeks postinterventions. One patient had premature atrial complexes on day 1 postprocedure with no subsequent CIED interrogation. The same patient had three subsequent angioplasty procedures with no reported ECG changes. The other patient had borderline ECG changes for lateral myocardial infarction 5 days postintervention. Both incidents were not related to device malfunction.
Even 2 weeks after the procedure, no procedure-related complication was documented. One of the patients developed hypotension 10 days after the angioplasty, and another patient had confusion; both were related to their underlying heart failure as documented by their primary physician.

Six-month primary patency was achieved in $62 \%(23 / 37)$ of the procedures. Ten patients (66\%) required an average of 1.4 reinterventions (range: 1-4 interventions) during the follow-up time with mean time to reintervention of 318 days (5-1,380 days). Two patients required early reinterventions within 10 days due to catheter dysfunction. A total of 14 patients (93\%) remain patent or with functional catheter at a mean follow-up time of 3.6 years (0.96-6 years). The last patient required fistula embolization due to failed recanalization of chronic occlusion of left subclavian vein.
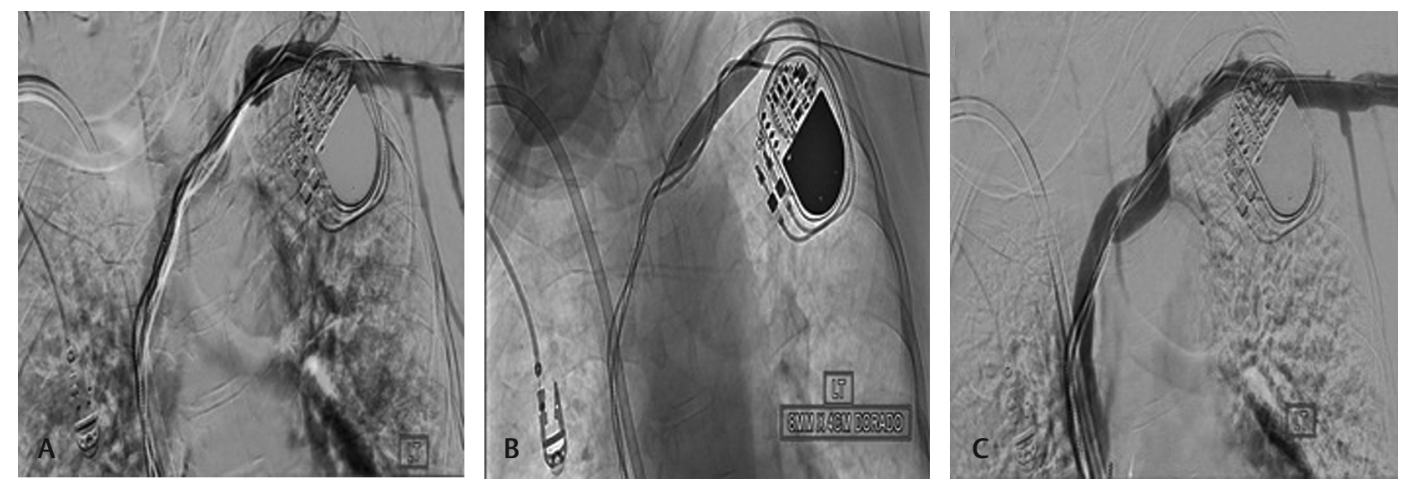

Fig. 1 (A) Focal stenosis at the subclavian vein at the point of lead insertion. (B) Balloon dilation with an $8 \times 40 \mathrm{~mm}$ balloon. (C) Interval improvement of the stenotic area following the dilation. 

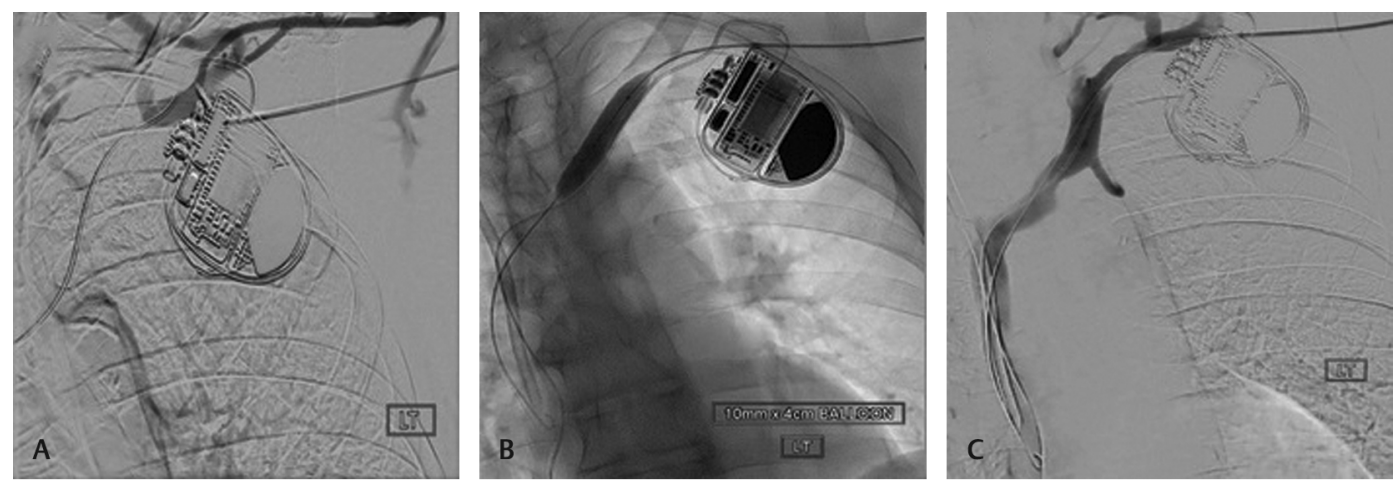

Fig. 2 (A) Another example of occluded subclavian vein where the lead was inserted. (B) Dilation with a $10 \times 40 \mathrm{~mm}$ balloon. (C) Postballoon angioplasty with complete opacification.

\section{Discussion}

Balloon angioplasty without stent placement is currently the preferred method for the management of central venous stenosis related to either CIED or hemodialysis catheters. ${ }^{5}$ Despite the concerns regarding the possible damage to CIED leads and further lead endothelialization, several studies have demonstrated the safety and effectiveness of balloon angioplasty across the CIED leads with no impact on wire integrity, position, or function on subsequent evaluation and lead interrogation. . $^{1,5,7,8,12,14}$ Asif et al reported the outcomes of balloon angioplasty in 28 hemodialysis patients with central venous stenosis due to CIED leads. Clinical success was achieved in all patients with no procedure-related complications. The primary and secondary patency rates were 9 and $86 \%$ at 12 months, respectively, requiring an average of 2.1 procedures/year. $^{7}$ In the present study, primary patency at 6 months was $62 \%$, and primary assisted patency without stent placement was $93 \%$ at a mean follow-up time of 3.6 years. In cases of stenoses refractory to balloon angioplasty, stent placement may be considered with or without lead extraction. Safe stent placement over CIED wires has been previously reported,, $911-15$ however, concerns remain regarding the long-term consequences of metallic stents on the function and integrity of entrapped CIED wires and the potential need to remove the leads in cases of malfunction and infection. Sotiriadis et al successfully treated five symptomatic patients with CEID-related CVO using sinus XL stents without lead extractions. ${ }^{6,14}$ Only one patient reported early battery dysfunction requiring replacement 2 years sooner than expected. ${ }^{14}$ Saad et al treated 14 patients with different stents and stent grafts across the lead wires with no complications related to device failure. ${ }^{12}$ In a pooled analysis of 104 patients who underwent management of CEID-related CVO, 25 patients were treated with stent placement jailing the leads in $72 \%(18 / 25)$ of patients, with no reports of any adverse outcomes within median follow-up time of 9.5 months. ${ }^{6}$ Other studies reported successful central venous recanalization and angioplasty with stent placement following lead extraction, ${ }^{1,8,10,16}$ however, this approach is more invasive and associated with higher costs. ${ }^{6,14}$ The American Society of Diagnostic and Interventional Nephrology recommends to avoid entrapment of CIED leads with stents, and to extract the leads whenever stent placement is deemed necessary. ${ }^{5}$

This study is limited by its small sample size, retrospective data collection, missing information on the date of pacemaker insertion, missing technical details related to balloon pressures and duration of inflation, and lack of systematic follow-up protocol and assessment of cardiac devices, including the scheduled CIED interrogation. However, findings of this study support the existing evidence on the safety and effectiveness of balloon angioplasty without lead extraction.

\section{Conflict of Interest}

None.

\section{References}

1 Sherk WM, Khaja MS, Good ED, Cunnane RT, Dasika NL, Williams DM. Hybrid venous recanalization and cardiac implantable electronic device lead revision procedures: a single-center retrospective analysis of 38 patients. Clin Imaging 2019;58:145-151

2 Abu-El-Haija B, Bhave PD, Campbell DN, et al. Venous stenosis after transvenous lead placement: a study of outcomes and risk factors in 212 consecutive patients. J Am Heart Assoc 2015;4(8):e001878

3 Oginosawa Y, Abe H, Nakashima Y.The incidence and risk factors for venous obstruction after implantation of transvenous pacing leads. Pacing Clin Electrophysiol 2002;25(11):1605-1611

4 Rozmus G, Daubert JP, Huang DT, Rosero S, Hall B, Francis C. Venous thrombosis and stenosis after implantation of pacemakers and defibrillators. J Interv Card Electrophysiol 2005;13(1):9-19

5 Saad TF, Hentschel DM, Koplan B, et al; ASDIN Clinical Practice Committee Workgroup. Cardiovascular implantable electronic device leads in CKD and ESRD patients: review and recommendations for practice. Semin Dial 2013;26(1):114-123

6 Riley RF, Petersen SE, Ferguson JD, Bashir Y. Managing superior vena cava syndrome as a complication of pacemaker implantation: a pooled analysis of clinical practice. Pacing Clin Electrophysiol 2010;33(4):420-425

7 Asif A, Salman L, Carrillo RG, et al. Patency rates for angioplasty in the treatment of pacemaker-induced central venous stenosis in hemodialysis patients: results of a multi-center study. Semin Dial 2009;22(6):671-676

8 Borsato GW, Rajan DK, Simons ME, Sniderman KW, Tan KT. Central venous stenosis associated with pacemaker leads: short-term results of endovascular interventions. J Vasc Interv Radiol 2012;23(3):363-367 
34 Central Venoplasty in Patient with Cardiac Pacemakers Aljarie et al.

9 Francis CM, Starkey IR, Errington ML, Gillespie IN. Venous stenting as treatment for pacemaker-induced superior vena cava syndrome. Am Heart J 1995;129(4):836-837

10 Klop B, Scheffer MG, McFadden E. Bracke F, van Gelder B. Treatment of pacemaker-induced superior vena cava syndrome by balloon angioplasty and stenting. Neth Heart J 2011;19(1):41-46

11 Lindsay HS, Chennells PM, Perrins EJ. Successful treatment by balloon venoplasty and stent insertion of obstruction of the superior vena cava by an endocardial pacemaker lead. Br Heart J 1994;71(4):363-365

12 Saad TF, Myers GR, Cicone J. Central vein stenosis or occlusion associated with cardiac rhythm management device leads in hemodialysis patients with ipsilateral arteriovenous access: a retrospective study of treatment using stents or stent-grafts. J Vasc Access 2010;11:293-302
13 Slonim SM, Semba CP, Sze DY, Dake MD. Placement of SVC stents over pacemaker wires for the treatment of SVC syndrome. J Vasc Interv Radiol 2000;11(2 Pt 1) :215-219

14 Sotiriadis C, Volpi S, Douek P, Chouiter A, Muller O, Qanadli SD. Are endovascular interventions for central vein obstructions due to cardiac implanted electronic devices effective? Front Surg 2018;5:49

15 Teo N, Sabharwal T, Rowland E, Curry P, Adam A. Treatment of superior vena cava obstruction secondary to pacemaker wires with balloon venoplasty and insertion of metallic stents. Eur Heart J 2002;23(18):1465-1470

16 Fu HX, Huang XM, Zhong L, et al. Outcome and management of pacemaker-induced superior vena cava syndrome. Pacing Clin Electrophysiol 2014;37(11):1470-1476 